\title{
Origin and evaluation of the four-spin operators in magnetic lattices
}

\author{
Carmen J. Calzado ${ }^{1, *}$ and Jean-Paul Malrieu ${ }^{2, \dagger}$ \\ ${ }^{1}$ Departamento de Química Física, Universidad de Sevilla, E-41012 Sevilla, Spain \\ ${ }^{2}$ Laboratoire de Physique Quantique, IRSAMC, Université Paul Sabatier, F-31062 Toulouse-Cedex, France
}

(Received 6 November 2003; revised manuscript received 12 January 2004; published 30 March 2004)

\begin{abstract}
The four-spin operators are suspected to have a significant amplitude and an impact on the collective properties in spin ladders and magnetic two-dimensional lattices. They originate from cyclic circulation of electrons (ring currents). Starting from a simple Hubbard Hamiltonian one may establish their form from a fourth order expansion of quasidegenerate perturbation theory. This form slightly deviates from the generally assumed biquadratic expression. From a quantitative point of view, their amplitude can be assessed from accurate $a b$ initio explicitly correlated wave functions and energies on embedded clusters. The present work shows that symmetry-broken density functional theory calculations may also provide estimates of the fourbody operator amplitudes, but reliable results require the use of a large Fock component in the exchange functional.
\end{abstract}

DOI: 10.1103/PhysRevB.69.094435

PACS number(s): 75.30.Et, 71.27.+a, 71.70.Gm, 75.50.Ee

\section{INTRODUCTION}

The Heisenberg Hamiltonians were introduced in the 1930 's ${ }^{1-3}$ to rationalize the properties of magnetic systems. This Hamiltonian can be derived as an effective Hamiltonian, working on the space generated by the products of the highest multiplicity ground state of the magnetic centers. It can be obtained from $\mathbb{N}$-electron Hamiltonians (either exact or simplified) through the perturbative expansion in the frame of the quasidegenerate perturbation theory (QDPT). The leading operators appear as first and second order contributions and concern the two-body effective exchange interactions $J_{i j}$ between the sites $i$ and $j$. The magnitude of these interactions decreases very rapidly as a function of the distance between the centers $i$ and $j$.

Strictly speaking, these terms are not unique, higher order corrections introduce four-spin and six-spin terms. The developments from simplified $\mathbb{N}$-electron Hamiltonians such as the Hubbard Hamiltonian for half-filled band, with one electron per site, show that the four-body operators are important in four-member rings. Respectively, the six-body terms play a role in the six-member rings. From a chemical point of view, these contributions can be related with the antiaromatic and aromatic character of the four-member and six-member rings, respectively. ${ }^{4}$ Similar derivations have been performed by solid state physicists. ${ }^{5}$ Recently these many-body effects have been suspected to play a role in the physics of spin lattices, involving square or rectangular plaquettes. ${ }^{6-13}$ For instance, their role has been invoked to resolve contradictions regarding the properties of two-leg ladders. ${ }^{12,13} \mathrm{Ab}$ initio quantum chemical calculations have provided direct estimates of the amplitude of these terms, which confirm their physical importance. ${ }^{14-16}$

Section II of the present work returns on the origin of the four-body corrections. Starting from a Hubbard Hamiltonian all the fourth order corrections involving circulation of electrons on the four-member rings are established. The resulting spin operators slightly deviate from the usually accepted biquadratic form of the Heisenberg Hamiltonian, although they coincide regarding the most important four-spin cyclic ex- change. This section also shows the possible occurrence of the four-body operators which involve hopping between second neighbor sites, i.e., those placed in the diagonal of the plaquette. Section III discusses the procedure to determine the amplitudes of the four-body corrections from $a b$ initio embedded cluster calculations. Previous works have used the eigenenergies and wave functions coming from extended configuration interaction (CI) calculations. ${ }^{14,16}$ The present work proposes a procedure to obtain an estimate of the fourbody terms from density functional theory (DFT) based symmetry-broken calculations, as frequently practiced for the two-body exchanges. As is well known, the quality of such estimates strongly depends on the exchange functional. ${ }^{17-21}$ As shown on the case of a series of spin ladders compounds and 2D cuprates, the mixing of the Fock and Slater exchanges which provides correct estimates of the first neighbor interactions happens to lead to values of the four-body terms which agree with the ab initio CI results.

\section{FORMAL ASPECTS}

\section{A. Formal requirements for a four-spin effective Hamiltonian}

The spin Hamiltonians, with respect to the exact Hamiltonian, must be considered as effective Hamiltonians, in the strict and rigorous definition of this concept. The effective Hamiltonians for a given $\mathbb{N}$-electron system work in a model space $\mathrm{S}$, the dimension of which, $N$, is much smaller than the Hilbert space relative to the exact Hamiltonian $H$. Calling $P_{\mathrm{S}}$ the projector on the model space

$$
H^{\mathrm{eff}}=P_{\mathrm{S}} H^{\mathrm{eff}} P_{\mathrm{S}} .
$$

The $N$ eigenvectors and eigenvalues of the effective Hamiltonian $H^{\text {eff }}$ fulfill two requirements: (i) the eigenenergies are the eigenenergies of $H$ and (ii) the eigenvectors are projections of the corresponding eigenvectors of $H$ onto the model space $\mathrm{S}$.

These $N$ eigenvectors of $H$ define a stable subspace, called the target space $\mathrm{S}^{\prime}$. Hence these $N$ eigenvectors $\psi_{m}$ of $H$ belonging to $\mathrm{S}^{\prime}$ : 
TABLE I. The effective spin Hamiltonian on the basis of the model space for $S_{z}=0 . \sum_{n n}$ refers to the sum over all the first-neighbor interactions $A=j_{a c}+j_{b d}+j_{a d}+j_{b c}, B=j_{a c}+j_{b d}+j_{a b}+j_{c d}$.

\begin{tabular}{cccccc}
\hline \hline$\left|a_{\downarrow} b_{\uparrow} c_{\downarrow} d_{\uparrow}\right|$ & $\left|a_{\uparrow} b_{\downarrow} c_{\uparrow} d_{\downarrow}\right|$ & $\left|a_{\downarrow} b_{\downarrow} c_{\uparrow} d_{\uparrow}\right|$ & $\left|a_{\uparrow} b_{\uparrow} c_{\downarrow} d_{\downarrow}\right|$ & $\left|a_{\downarrow} b_{\uparrow} c_{\uparrow} d_{\downarrow}\right|$ & $\left|a_{\uparrow} b_{\downarrow} c_{\downarrow} d_{\uparrow}\right|$ \\
\hline$-\Sigma j_{n n}-h_{1}$ & & & & \\
$h_{1}$ & $-\Sigma j_{n n}-h_{1}$ & & & \\
$j_{b c}$ & $j_{a d}$ & $-A-h_{2}$ & & \\
$j_{a d}$ & $j_{b c}$ & $h_{2}$ & $-A-h_{2}$ & & \\
$j_{c d}$ & $j_{a b}$ & $j_{b d}$ & $j_{a c}$ & $-B-h_{3}$ & \\
$j_{a b}$ & $j_{c d}$ & $j_{a c}$ & $j_{b d}$ & $h_{3}$ & $-B-h_{3}$ \\
\hline \hline
\end{tabular}

$$
H\left|\psi_{m}\right\rangle=E_{m}\left|\psi_{m}\right\rangle
$$

define entirely the effective Hamiltonian $H^{\text {eff }}$ by the conditions

$$
H^{\mathrm{eff}}\left|P_{\mathrm{S}} \psi_{m}\right\rangle=E_{m}\left|P_{\mathrm{S}} \psi_{m}\right\rangle, \quad \forall \quad \psi_{m} \in \mathrm{S}^{\prime} .
$$

This basic equation leads to the spectral definition of the Bloch effective Hamiltonian ${ }^{22}$

$$
H_{\mathrm{Bloch}}^{\mathrm{eff}}=\sum_{m=1, N}\left|P_{\mathrm{S}} \psi_{m}\right\rangle E_{m}\left\langle P_{\mathrm{S}} \psi_{m}^{\perp}\right|
$$

where $\left|P_{\mathrm{S}} \psi_{m}^{\perp}\right\rangle$ represents the biorthogonal vector associated to $\left|P_{\mathrm{S}} \psi_{m}\right\rangle$. Actually the projections of the (orthogonal) eigenvectors of $H$ onto the model space have in general no reason to be orthogonal. They define an overlap matrix $S$ :

$$
S_{m n}=\left\langle P_{\mathrm{S}} \psi_{m} \mid P_{\mathrm{S}} \psi_{n}\right\rangle
$$

and

$$
P_{\mathrm{S}} \psi_{m}^{\perp}=S^{-1} P_{\mathrm{S}} \psi_{m}
$$

The Bloch effective Hamiltonian is non-Hermitian. Its $N^{2}$ matrix elements are defined from the $N^{2}$ conditions imposed by Eq. (4).

An Hermitian definition of the effective Hamiltonian was proposed a few years later by des Cloizeaux. ${ }^{23}$ Orthogonalizing the projected eigenvectors $\left|P_{\mathrm{S}} \psi_{m}\right\rangle$ through a leastmoving $S^{-1 / 2}$ transformation

$$
\left\{\psi_{m}^{\prime}\right\}=S^{-1 / 2}\left\{P_{\mathrm{S}} \psi_{m}\right\}
$$

the des Cloizeaux effective Hamiltonian is such that

$$
\begin{gathered}
H_{d C}^{\mathrm{eff}}\left|\psi_{m}^{\prime}\right\rangle=E_{m}\left|\psi_{m}^{\prime}\right\rangle, \\
H_{d C}^{\mathrm{eff}}=\sum_{m=1, N}\left|\psi_{m}^{\prime}\right\rangle E_{m}\left\langle\psi_{m}^{\prime}\right| .
\end{gathered}
$$

The spin Hamiltonians concern half-filled bands. For sites with spin $1 / 2$ the model space is spanned by the neutral valence-bond determinants, i.e., by those where each site bears one and only one electron. These determinants all have the same space part and only differ by the spin distributions. The resulting effective Hamiltonian therefore appears as a spin-only Hamiltonian. For a $S_{z}=02 n$-site problem the dimension of the model space is $\mathbb{C}_{2 n}^{n}$ and the target space is spanned by the $\mathrm{C}_{2 n}^{n}$ eigenstates of $H$ having the largest projections on the model space. For the magnetic regime of interactions the target states are usually the lowest eigenvectors of $H$.

From these basic considerations one can see that a spin Hamiltonian has no reason to be reducible to two-body operators, as usually assumed in Heisenberg Hamiltonians

$$
H_{\mathrm{Heis}}=\sum_{\langle i j\rangle} J_{i j} \overrightarrow{S_{i}} \vec{S}_{j}
$$

They in principle will present four-body, six-body,... , operators, since the matrix element of $H^{\text {eff }}$ between two spin distributions $\phi_{I}$ and $\phi_{J}$ differing by four, six, ..., spins has no reason to be zero:

$$
\left\langle\phi_{I}\left|H^{\mathrm{eff}}\right| \phi_{J}\right\rangle=\sum_{m=1, N}\left\langle\phi_{I} \mid \psi_{m}^{\prime}\right\rangle E_{m}\left\langle\psi_{m}^{\prime} \mid \phi_{J}\right\rangle
$$

The simplest approach to establish the structure of an Heisenberg Hamiltonian starts from a Hubbard single band Hamiltonian. The metallic on-site singly occupied orbitals $i, j, \ldots$, are supposed to have been optimized, including variational delocalization tails on the ligands surrounding the metal ions. In this Hamiltonian

$$
\begin{aligned}
H_{\mathrm{Hubb}}= & \sum_{\langle i j\rangle} t_{i j}\left(a_{i \uparrow}^{\dagger} a_{j \uparrow}+a_{j \uparrow}^{\dagger} a_{i \uparrow}+a_{i \downarrow}^{\dagger} a_{j \downarrow}+a_{j \downarrow}^{\dagger} a_{i \downarrow}\right) \\
& +U \sum_{i} n_{i \uparrow} n_{i \downarrow}
\end{aligned}
$$

the $t$ integrals are intersite hopping integrals and $U$ is the on-site Coulomb repulsion. The neutral valence-bond determinants have an energy zero, the singly ionic ones have an

TABLE II. The effective spin Hamiltonian on the basis of the model space for $S_{z}=1$. The term $-\Sigma_{i} j_{a i}^{\prime}$ corresponds to $-j_{a b}^{\prime}$ $-j_{a c}^{\prime}-j_{a d}^{\prime}$, and similarly for the rest.

\begin{tabular}{cccc}
\hline \hline$\left|a_{\downarrow} b_{\uparrow} c_{\uparrow} d_{\uparrow}\right|$ & $\left|a_{\uparrow} b_{\downarrow} c_{\uparrow} d_{\uparrow}\right|$ & $\left|a_{\uparrow} b_{\uparrow} c_{\downarrow} d_{\uparrow}\right|$ & $\left|a_{\uparrow} b_{\uparrow} c_{\uparrow} d_{\downarrow}\right|$ \\
\hline$-\Sigma_{i} j_{a i}^{\prime}$ & & & \\
$j_{a b}^{\prime}$ & $-\Sigma_{i} j_{b i}^{\prime}$ & & \\
$j_{a c}^{\prime}$ & $j_{b c}^{\prime}$ & $-\Sigma_{i} j_{c i}^{\prime}$ & \\
$j_{a d}^{\prime}$ & $j_{b d}^{\prime}$ & $j_{c d}^{\prime}$ & $-\Sigma_{i} j_{d i}^{\prime}$ \\
\hline \hline
\end{tabular}




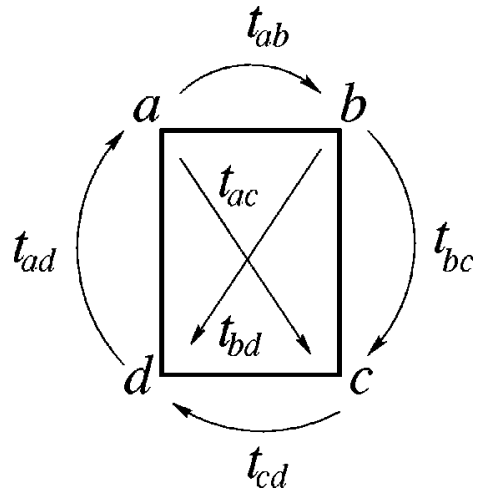

FIG. 1. First and second-neighbor hopping integrals in a plaquette.

energy $U$. The second order expression of the intersite effective exchange can be obtained by using the quasidegenerate perturbation theory

$$
J_{i j}=\frac{4 t_{i j}^{2}}{U} .
$$

Let us consider now a four-site problem. The model space is six dimensional for $S_{z}=0$, spanned by determinants which may be associated two by two:

$$
\begin{aligned}
& \phi_{1}=|a(\downarrow) b(\uparrow) c(\downarrow) d(\uparrow)|, \quad \phi_{1}^{\prime}=|a(\uparrow) b(\downarrow) c(\uparrow) d(\downarrow)|, \\
& \phi_{2}=|a(\downarrow) b(\downarrow) c(\uparrow) d(\uparrow)|, \quad \phi_{2}^{\prime}=|a(\uparrow) b(\uparrow) c(\downarrow) d(\downarrow)|, \\
& \phi_{3}=|a(\uparrow) b(\downarrow) c(\downarrow) d(\uparrow)|, \quad \phi_{3}^{\prime}=|a(\downarrow) b(\uparrow) c(\uparrow) d(\downarrow)| .
\end{aligned}
$$

They generate one quintet, three triplet, and two singlet states. For $S_{z}=1$, the four determinants $\quad|a(\downarrow) b(\uparrow) c(\uparrow) d(\uparrow)|, \quad|a(\uparrow) b(\downarrow) c(\uparrow) d(\uparrow)|$, $|a(\uparrow) b(\uparrow) c(\downarrow) d(\uparrow)|$, and $|a(\uparrow) b(\uparrow) c(\uparrow) d(\downarrow)|$ generate three triplet and one quintet states. In a six-dimensional space an Hermitian effective Hamiltonian may in principle have $[(36+6) / 2]=21,\left\{\left(\left[\mathbb{C}_{2 n}^{n}\right]^{2}+\mathbb{C}_{2 n}^{n}\right) / 2\right\}$, different matrix elements. However it must obey symmetry constraints and the eigenvectors have to commute with $S^{2}$. The number of degrees of freedom is much lower. By imposing the zero of energy to the upper multiplet state, the sum of the matrix elements on each line (or column) is zero. There are five eigenenergy differences, only one degree of freedom in the singlet eigenvectors and three in the triplet eigenvectors, i.e., nine degrees of freedom. The effective Hamiltonian matrix for $S_{z}=0$ will take the form shown in Table I. The $j_{m n}$ and $h_{p}$ parameters concern, respectively, two-body spin exchange operators and four-body operators. For $S_{z}=1$ the effective Hamiltonian matrix takes the form shown in Table II. The spin algebra imposes some relations between $j_{m n}, j_{m n}^{\prime}$, and $h_{p}$ quantities.

\section{B. Perturbative derivation}

While the two-body operators appear at second order of the quasidegenerated perturbation theory (QDPT), it is necessary to go to fourth-order to permute four spins. Let us a)

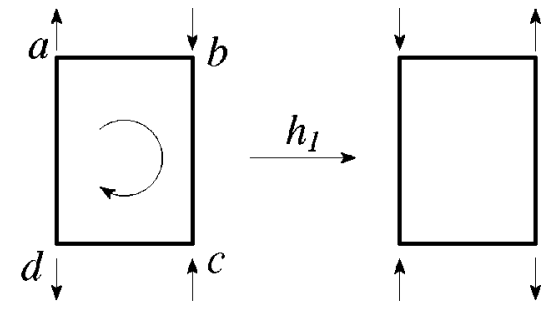

b)

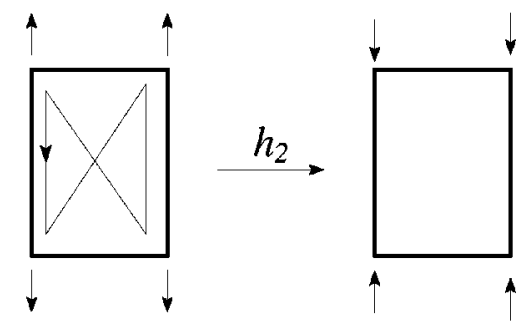

c)

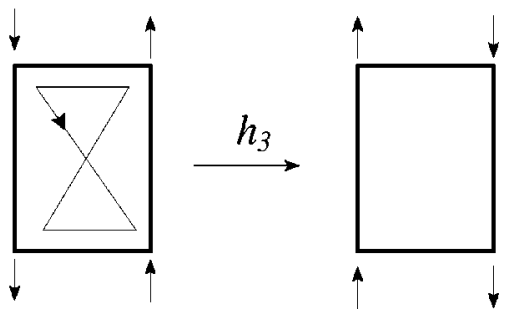

FIG. 2. The three four-body terms: (a) circular movement of the electrons $h_{1}$, (b) simultaneous exchange along the legs $h_{2}$, and (c) simultaneous exchange across the rungs $h_{3}$.

recall the expression of the fourth order contribution to the effective Hamiltonian. If $I, J, K, L$ represent vectors of the model space, $\alpha, \beta, \gamma$ being vectors of the outer space,

$$
\begin{aligned}
\left\langle I\left|H^{(4)}\right| J\right\rangle= & \sum_{\alpha, \beta, \gamma} \frac{\langle I|V| \alpha\rangle\langle\alpha|V| \beta\rangle\langle\beta|V| \gamma\rangle\langle\gamma|V| J\rangle}{\Delta \alpha \Delta \beta \Delta \gamma} \\
& -\sum_{\alpha, \beta, K} \frac{\langle I|V| \alpha\rangle\langle\alpha|V| \beta\rangle\langle\beta|V| K\rangle\langle K|V| J\rangle}{\Delta \alpha^{2} \Delta \beta} \\
& +\sum_{\alpha, \beta, K} \frac{\langle I|V| \alpha\rangle\langle\alpha|V| K\rangle\langle K|V| \beta\rangle\langle\beta|V| J\rangle}{\Delta \alpha^{2} \Delta \beta} \\
& +\sum_{\alpha, K, L} \frac{\langle I|V| \alpha\rangle\langle\alpha|V| K\rangle\langle K|V| L\rangle\langle L|V| J\rangle}{\Delta \alpha^{3}}
\end{aligned}
$$

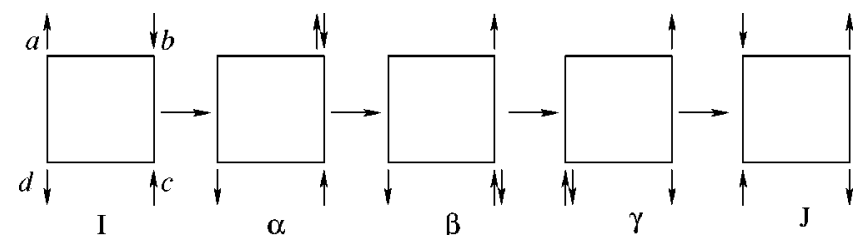

FIG. 3. Contribution to $h_{1}$ due to the cyclic circulation of the four electrons of the plaquette. 

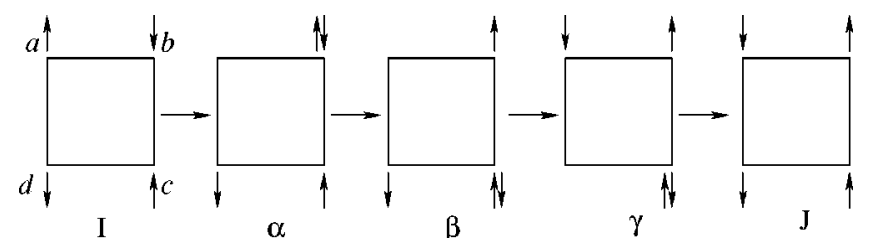

FIG. 4. Cyclic contributions to the exchange of two spins on the same bond.

where $\Delta \alpha$ represent the zero-order energy differences $E_{0}^{0}$ $-E_{\alpha}^{0}$.

Let consider now a four-atom ring (Fig. 1), with $t_{a b}, t_{b c}$, $t_{c d}$, and $t_{a d}$ being hopping integrals (the $t_{a c}$ and $t_{b d}$ integrals being zero). We can go from $|a(\uparrow) b(\downarrow) c(\uparrow) d(\downarrow)|$ to $|a(\downarrow) b(\uparrow) c(\downarrow) d(\uparrow)|$ through a cyclic circulation of electrons [Fig. 2(a)], for instance, as in Fig. 3, which will lead to a contribution to $h_{1}$ equal to

$$
g_{a b c d}=\frac{t_{a b} t_{b c} t_{c d} t_{d a}}{U^{3}} .
$$

The possible contributions are numerous and lead to

$$
h_{1}=40 \frac{t_{a b} t_{b c} t_{c d} t_{d a}}{U^{3}}=40 g_{a b c d} .
$$

However, it should be noticed that such cyclic fourth order corrections also appear between determinants which differ only by two spins, either on a bond, for instance, from $|a(\uparrow) b(\downarrow) c(\uparrow) d(\downarrow)|$ to $|a(\downarrow) b(\uparrow) c(\uparrow) d(\downarrow)|$ as in Fig. 4, or between nonbonded atoms, for instance, from $|a(\uparrow) b(\downarrow) c(\downarrow) d(\uparrow)|$ to $|a(\uparrow) b(\uparrow) c(\downarrow) d(\downarrow)|$ as in Fig. 5. Diagonal corrections also exist, for instance as in Fig. 6.

The final fourth order contributions may be written in a matrix form in terms of $g_{a b c d}$ quantities. Let us call $\mathrm{O}_{\text {per } 4(0)}^{a b c d}$ the operator for $S_{z}=0$ and $\mathbb{O}_{\text {per4(1) }}^{a b c d}$ its $S_{z}=1$ counterpart. A careful and exhaustive counting of all cyclic corrections lead to the matrix representation of the four-body operators reported in Tables III and IV.

The coupling between $|a(\uparrow) b(\downarrow) c(\uparrow) d(\downarrow)|$ and $|a(\downarrow) b(\uparrow) c(\downarrow) d(\uparrow)|$ which is the largest term $\left(h_{1} a_{a \downarrow}^{\dagger} a_{b \uparrow}^{\dagger} a_{c \downarrow}^{\dagger} a_{d \uparrow}^{\dagger} a_{d \downarrow} a_{c \uparrow} a_{b \downarrow} a_{a \uparrow}=40 g_{a b c d}\right)$ commutes four spins. The other terms only exchange two spins but they only act in presence of two other spins in the plaquette. For instance, in the coupling between $|a(\uparrow) b(\downarrow) c(\uparrow) d(\downarrow)|$ and $|a(\downarrow) b(\uparrow) c(\uparrow) d(\downarrow)|,\left(-12 g_{a b c d} a_{a \uparrow}^{\dagger} a_{b \downarrow}^{\dagger} a_{c \uparrow}^{\dagger} a_{d \downarrow}^{\dagger} a_{d \downarrow} a_{c \uparrow} a_{b \uparrow} a_{a \downarrow}\right)$, the spins $c$ and $d$ appear as spectators, but this correction to the exchange between $a$ and $b$ only exists if $a$ and $b$ belong

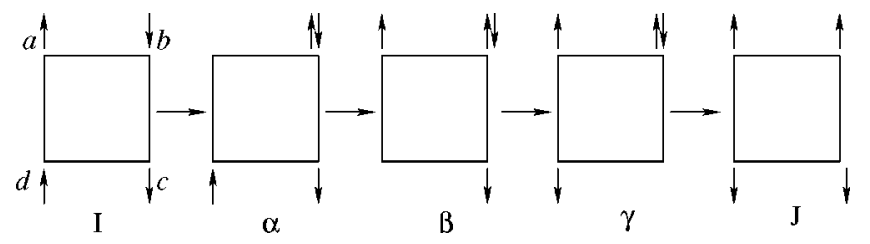

FIG. 5. Cyclic contributions to the exchange between two nonbonded atoms.

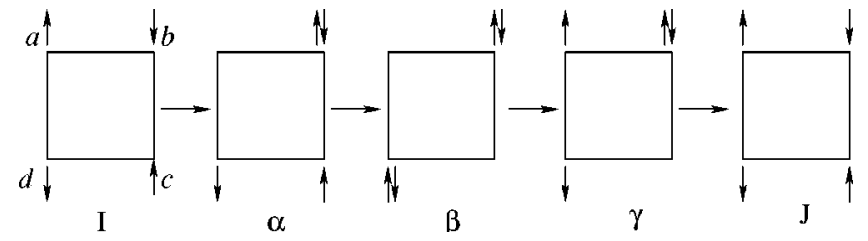

FIG. 6. Cyclic correction to the diagonal energy.

to a ring, that is, it is a four-body correction to the two-body operator, which results from the electronic circulation along the ring.

Notice that the fourth order correction linked to the cyclic circulation on the ring does not bring any contribution to the $h_{2}$ and $h_{3}$ terms, i.e., to the interaction between $|a(\uparrow) b(\uparrow) c(\downarrow) d(\downarrow)|$ and $|a(\downarrow) b(\downarrow) c(\uparrow) d(\uparrow)|[$ Fig. 2(b)] or between $|a(\uparrow) b(\downarrow) c(\downarrow) d(\uparrow)| \quad$ and $|a(\downarrow) b(\uparrow) c(\uparrow) d(\downarrow)|$ [Fig. 2(c)] because we have imposed $t_{a c}=t_{b d}=0$. It is possible to check that the $O_{\text {per4(S) }}^{a b c d}$ operators commute with $S^{2}$ and that their eigenenergies for the triplet and quintet states are equal for $S_{z}=0$ and $S_{z}=1$.

\section{Comparison with biquadratic spin operators}

It is worth comparing the so-obtained four-body corrections to the classical expression in terms of biquadratic spin operators for a ring

$$
\begin{aligned}
0_{\text {biqua }}^{a b c d}= & J_{\text {ring }}^{a b c d}\left[\left(\overrightarrow{S_{a}} \overrightarrow{S_{b}}\right)\left(\overrightarrow{S_{c}} \overrightarrow{S_{d}}\right)+\left(\overrightarrow{S_{a}} \overrightarrow{S_{d}}\right)\left(\overrightarrow{S_{b}} \overrightarrow{S_{c}}\right)\right. \\
& \left.-\left(\overrightarrow{S_{a}} \overrightarrow{S_{c}}\right)\left(\overrightarrow{S_{b}} \overrightarrow{S_{d}}\right)-\frac{1}{16}\right] .
\end{aligned}
$$

The constant $1 / 16$ has been introduced to put to zero the energy corrections on the quintet state. For the same reason, the two-body Heisenberg Hamiltonian is then shifted from its usual form and becomes

$$
H_{\mathrm{Heis}}=\sum_{\langle i j\rangle} J_{i j}\left(\vec{S}_{i} \vec{S}_{j}-\frac{1}{4}\right) .
$$

The term $J_{\text {ring }}^{a b c d}$ has been established to be $80 t^{4} / U^{3}$ for a square plaquette. ${ }^{4}$ More generally it is possible to write

$$
J_{\text {ring }}^{a b c d}=80 t_{a b} t_{b c} t_{c d} t_{a d} / U^{3}=80 g_{a b c d} .
$$

The matrix representation of this operator in $g_{a b c d}$ units for $S_{z}=0$ is shown in Table V and in Table VI for $S_{z}=1$.

$$
\text { The four-spin permuting operator }
$$
$|a(\downarrow) b(\uparrow) c(\downarrow) d(\uparrow)\rangle\langle a(\uparrow) b(\downarrow) c(\uparrow) d(\downarrow)|$, which is the largest one, coincide with the exact perturbative value $\left(40 g_{a b c d}\right)$. The deviations on the off-diagonal elements $\left( \pm 2 g_{a b c d}\right)$ are small, but they result in more significant deviations on the diagonal $\left(8 g_{a b c d}\right.$ for $\left.S_{z}=0\right)$. Hence the fourth order corrections do not coincide with the usual formulation. It can be shown that the operator strictly derived from the fourth order perturbative expansion can be written as

$$
\mathrm{O}_{\text {per4 }}^{a b c d}=\mathrm{O}_{\text {biqua }}^{a b c d}+\mathrm{O}^{\prime}
$$


TABLE III. Matrix representation of $\mathbb{O}_{\text {per } 4(0)}^{a c d}$ operator for $S_{z}=0$ in the perturbation-based effective Hamiltonian, in terms of $g_{a b c d}$ units.

\begin{tabular}{|c|c|c|c|c|c|c|}
\hline & $\left|a_{\downarrow} b_{\uparrow} c_{\downarrow} d_{\uparrow}\right|$ & $\left|a_{\uparrow} b_{\downarrow} c_{\uparrow} d_{\downarrow}\right|$ & $\left|a_{\downarrow} b_{\downarrow} c_{\uparrow} d_{\uparrow}\right|$ & $\left|a_{\uparrow} b_{\uparrow} c_{\downarrow} d_{\downarrow}\right|$ & $\left|a_{\downarrow} b_{\uparrow} c_{\uparrow} d_{\downarrow}\right|$ & $\mid a_{\uparrow} b_{\downarrow} c_{\downarrow} d_{\uparrow}$ \\
\hline \multirow{6}{*}{$O_{\operatorname{per} 4(0)}^{a b c d}$} & 8 & & & & & \\
\hline & 40 & 8 & & & & \\
\hline & -12 & -12 & 8 & & & \\
\hline & -12 & -12 & 0 & 8 & & \\
\hline & -12 & -12 & 8 & 8 & 8 & \\
\hline & -12 & -12 & 8 & 8 & 0 & 8 \\
\hline
\end{tabular}

where the operator $O^{\prime}$ is

$$
\begin{aligned}
\mathrm{O}^{\prime}= & -\frac{J_{\text {ring }}^{a b c d}}{10}\left[\left(\overrightarrow{S_{a}} \overrightarrow{S_{b}}-\frac{1}{4}\right) S_{c} S_{d}+\left(\overrightarrow{S_{a}} \overrightarrow{S_{c}}-\frac{1}{4}\right) S_{b} S_{d}\right. \\
& +\left(\overrightarrow{S_{a}} \vec{S}_{d}-\frac{1}{4}\right) S_{b} S_{c}+\left(\overrightarrow{S_{b}} \overrightarrow{S_{c}}-\frac{1}{4}\right) S_{a} S_{d} \\
& \left.+\left(\overrightarrow{S_{b}} \overrightarrow{S_{d}}-\frac{1}{4}\right) S_{a} S_{c}+\left(\overrightarrow{S_{c}} \vec{S}_{d}-\frac{1}{4}\right) S_{a} S_{b}\right]
\end{aligned}
$$

and $S_{a}=S_{b}=S_{c}=S_{d}=1 / 2$ are the local spin momentum. This difference between the two formulations results in a correction to the two-body interaction values

$$
J_{\text {Heis }}=\frac{4 t^{2}}{U}-\frac{4 t^{4}}{U^{3}}=J_{\text {pert }}-\frac{J_{\text {ring }}^{a b c d}}{20} .
$$

Now if the $t_{a c}$ and $t_{b d}$ hopping integrals are not negligible, one should consider as well the circuits

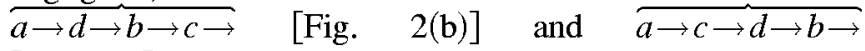
[Fig. 2(c)]. They will generate similar corrections in terms of quantities $g_{a d b c}=t_{a d} t_{b d} t_{b c} t_{c a} / U^{3}$ and $g_{a c d b}=t_{a c} t_{c d} t_{b d} t_{a b} /$ $U^{3}$ which contribute to $\mathbb{O}_{(S)}^{a c b d}$ and $\mathbb{O}_{(S)}^{a d b c}$ operators, where $S$ is the absolute value of $S_{z}(0$ or 1$)$.

The four-spin exchanges which commute couples of $\alpha$ and $\beta$ pairs of spins on opposite bonds, for instance, $\quad|a(\downarrow) b(\downarrow) c(\uparrow) d(\uparrow)| \quad$ into $|a(\uparrow) b(\uparrow) c(\downarrow) d(\downarrow)|$ through the $a \rightarrow d \rightarrow b \rightarrow c \rightarrow$ circulation, $h_{2}$, will have a $40 g_{a d b c}$ amplitude. The $h_{3}$ operator will similarly have an amplitude $40 g_{a c d b}$. Of course the $\overparen{a \rightarrow d \rightarrow b \rightarrow c \rightarrow}$ and $\overparen{a \rightarrow c \rightarrow d \rightarrow b \rightarrow}$ cyclic terms also affect the two-body spin

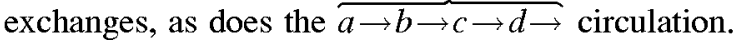

TABLE IV. Matrix representation of the $0_{\text {per4(1) }}^{a b c d}$ operator for $S_{z}=1$ in the perturbation-based effective Hamiltonian, in terms of $g_{a b c d}$ units.

\begin{tabular}{ccccc}
\hline \hline & $\left|a_{\downarrow} b_{\uparrow} c_{\uparrow} d_{\uparrow}\right|$ & $\left|a_{\uparrow} b_{\downarrow} c_{\uparrow} d_{\uparrow}\right|$ & $\left|a_{\uparrow} b_{\uparrow} c_{\downarrow} d_{\uparrow}\right|$ & $\left|a_{\uparrow} b_{\uparrow} c_{\uparrow} d_{\downarrow}\right|$ \\
\hline $\mathrm{O}_{\mathrm{per} 4(1)}^{a b c d}$ & -4 & & & \\
& 8 & -4 & & \\
& -12 & 8 & -4 & \\
8 & -12 & 8 & -4 \\
\hline \hline
\end{tabular}

For a general cluster, the total four-body operator will be the sum of the three four-body operators corresponding to the three possible four-step circulations along the edges of a (possibly irregular) tetrahedron ${ }^{24}$

$$
\mathrm{O}^{[a b c d]}=\mathrm{O}^{a b c d}+\mathrm{O}^{a d b c}+\mathrm{O}^{a c d b} .
$$

The extended Heisenberg Hamiltonian can be written as $^{9-11,16}$

$$
\begin{aligned}
H_{\text {Heis }}^{\text {biqua }}= & \sum_{\langle i j\rangle} J_{i j}\left(\overrightarrow{S_{i}} \vec{S}_{j}-\frac{1}{4}\right)+\sum_{\langle i j k l\rangle} J_{\text {ring }}^{i j k l}\left[\left(\overrightarrow{S_{i}} \overrightarrow{S_{j}}\right)\left(\overrightarrow{S_{k}} \overrightarrow{S_{l}}\right)\right. \\
& \left.+\left(\overrightarrow{S_{i}} \overrightarrow{S_{l}}\right)\left(\overrightarrow{S_{j}} \overrightarrow{S_{k}}\right)-\left(\overrightarrow{S_{i}} \overrightarrow{S_{k}}\right)\left(\overrightarrow{S_{j}} \overrightarrow{S_{l}}\right)-\frac{1}{16}\right]
\end{aligned}
$$

where $J_{i j}$ corresponds to the nearest-neighbor exchange or the second-neighbor exchange depending on the relative position of $i$ and $j$ and $J_{\text {ring }}^{i j k l}$ represents the three types of fourbody cyclic interactions: $J_{\text {ring }}^{a b c d}=2 h_{1}, \quad J_{\text {ring }}^{a d b c}=2 h_{2}$, and $J_{\text {ring }}^{a c d b}=2 h_{3}$. The matrix representation of this extended Heisenberg Hamiltonian, involving two-body and four-body terms is shown in Table VII.

Similarly, the perturbation-based Hamiltonian can be written as

$$
\begin{aligned}
H_{\text {Heis }}^{\text {per4 }}= & \sum_{\langle i j\rangle} J_{i j}\left(\overrightarrow{S_{i}} \vec{S}_{j}-\frac{1}{4}\right)+\sum_{\langle i j k l\rangle} J_{\text {ring }}^{i j k l}\left[\left(\overrightarrow{S_{i}} \overrightarrow{S_{j}}\right)\left(\overrightarrow{S_{k}} \vec{S}_{l}\right)\right. \\
& \left.+\left(\overrightarrow{S_{i}} \overrightarrow{S_{l}}\right)\left(\overrightarrow{S_{j}} \overrightarrow{S_{k}}\right)-\left(\overrightarrow{S_{i}} \overrightarrow{S_{k}}\right)\left(\overrightarrow{S_{j}} \overrightarrow{S_{l}}\right)-\frac{1}{16}\right] \\
& -\sum_{i j k l} \frac{1}{10}\left(J_{\text {ring }}^{i j k l}+J_{\text {ring }}^{i k j l}+J_{\text {ring }}^{i l j k}\right)\left(\vec{S}_{i} \vec{S}_{j}-\frac{1}{4}\right) S_{k} S_{l} .
\end{aligned}
$$

The matrix representation of this perturbation-based Hamiltonian is shown in Table VIII. Comparing Tables VII and VIII makes clear the difference between the two formalisms.

In the case of a rectangle, with $t_{\|}$and $t_{\perp}$ on the external bonds and $t_{d}$ on the diagonal of the rectangle (as occurs in ladder cuprates) 
TABLE V. Matrix representation of the biquadratic operator in the Heisenberg extended matrix for $S_{z}$ $=0$ in $g_{a b c d}$ units.

\begin{tabular}{|c|c|c|c|c|c|c|}
\hline & $\left|a_{\downarrow} b_{\uparrow} c_{\downarrow} d_{\uparrow}\right|$ & $\left|a_{\uparrow} b_{\downarrow} c_{\uparrow} d_{\downarrow}\right|$ & $\left|a_{\downarrow} b_{\downarrow} c_{\uparrow} d_{\uparrow}\right|$ & $\left|a_{\uparrow} b_{\uparrow} c_{\downarrow} d_{\downarrow}\right|$ & $\left|a_{\downarrow} b_{\uparrow} c_{\uparrow} d_{\downarrow}\right|$ & $\left|a_{\uparrow} b_{\downarrow} c_{\downarrow} d_{\uparrow}\right|$ \\
\hline \multirow[t]{6}{*}{$\mathrm{O}_{\text {biqua }(0)}^{a b c d}$} & 0 & & & & & \\
\hline & 40 & 0 & & & & \\
\hline & -10 & -10 & 0 & & & \\
\hline & -10 & -10 & 0 & 0 & & \\
\hline & -10 & -10 & 10 & 10 & 0 & \\
\hline & -10 & -10 & 10 & 10 & 0 & 0 \\
\hline
\end{tabular}

$$
\begin{aligned}
& g_{a b c d}=\frac{\left(t_{\|} t_{\perp}\right)^{2}}{U^{3}}, \\
& g_{a d b c}=\frac{\left(t_{\|} t_{d}\right)^{2}}{U^{3}}, \\
& g_{a c d b}=\frac{\left(t_{\perp} t_{d}\right)^{2}}{U^{3}} .
\end{aligned}
$$

As a result one sees that

$$
\begin{aligned}
& \frac{h_{2}}{h_{1}}=\frac{g_{a d b c}}{g_{a b c d}}=\left(\frac{t_{d}}{t_{\|}}\right)^{2}=\frac{J_{d}}{J_{\|}}, \\
& \frac{h_{3}}{h_{1}}=\frac{g_{a c b d}}{g_{a b c d}}=\left(\frac{t_{d}}{t_{\perp}}\right)^{2}=\frac{J_{d}}{J_{\perp}} .
\end{aligned}
$$

These relations are also valid for the canonical biquadratic expression of the four-body effects. All these developments have been obtained from the Hubbard Hamiltonian. Ab initio calculations, which involve more physical effects, may in principle lead to values of the four-body effects deviating from the above relations.

\section{NUMERICAL EVALUATION OF THE FOUR-BODY OPERATORS FROM AB INITIO CALCULATIONS ON PLAQUETTES}

The amplitudes of the two-body exchange operators $J$ are usually obtained from energy differences on two-site fragments, properly embedded in the Madelung field. When $a b$ initio CI calculations are performed, the magnetic coupling is obtained as the energy difference between $S^{2}$ eigenfunctions. For instance, for two centers with $S_{z}=1 / 2, J$ is equal to the

TABLE VI. Matrix representation of the biquadratic operator in the extended Heisenberg matrix for $S_{z}=1$, in $g_{a b c d}$ units.

\begin{tabular}{ccccc}
\hline \hline & $\left|a_{\downarrow} b_{\uparrow} c_{\uparrow} d_{\uparrow}\right|$ & $\left|a_{\uparrow} b_{\downarrow} c_{\uparrow} d_{\uparrow}\right|$ & $\left|a_{\uparrow} b_{\uparrow} c_{\downarrow} d_{\uparrow}\right|$ & $\left|a_{\uparrow} b_{\uparrow} c_{\uparrow} d_{\downarrow}\right|$ \\
\hline $0_{\text {biqua(1) }}^{a b c d}$ & -10 & & & \\
& 10 & -10 & & \\
& -10 & 10 & -10 & -10 \\
\hline \hline
\end{tabular}

energy difference between the lowest singlet and triplet states.

Density functional theory uses an unrestricted formalism and performs a calculation of the highest $S_{z}$ component of the upmost multiplet and a symmetry-broken calculation for the lower value of $S_{z}$, which is a mixture of spin eigenstates. Approximated projections of the low spin determinant provide values of the coupling constant. ${ }^{25}$

\section{A. Ab initio calculations}

Performing $a b$ initio CI calculations on four-site fragments make possible direct and simultaneous determination of the amplitude of the first- and second-neighbor two-body exchanges, as well as of the four-body operators. This determination proceeds through the use of the effective Hamiltonian theory according to the formalism of $\mathrm{Bloch}^{22}$ or des Cloizeaux $^{23}$ and implies the knowledge of the exact energies and projections of the eigenfunction on the model space.

For a general case of an arbitrary irregular tetrahedron one may always extract the amplitude of the six two-body operators and the three terms of the four-body operators from the knowledge of the five energy differences between the quintet, three triplet and the two singlet states, and the degrees of freedom in the coefficients of the wave functions, namely, three for the triplet manifold and one for the singlet states. Actually, looking, for instance, at the $S_{z}=1$ triplet which has expanded on four determinants, the orthogonality with the quintet state and the normalization leave only two degrees of freedom for the lowest triplet state. The second triplet must be also orthogonal to the first one, reducing its degrees of freedom to one, and the third one is entirely determined by the orthogonality to the other two triplet states and the quintet. A similar rationalization is possible in the singlet manifold.

The theoretical procedure has been explicited elsewhere $^{14,15}$ and a systematic study of three spin ladders $\left(\mathrm{SrCu}_{2} \mathrm{O}_{3}, \mathrm{CaCu}_{2} \mathrm{O}_{3}\right.$, and $\left.\mathrm{Sr}_{2} \mathrm{Cu}_{3} \mathrm{O}_{5}\right)$ and of the $2 \mathrm{D}$ square spin lattice $\mathrm{La}_{2} \mathrm{CuO}_{4}$ has been recently reported. ${ }^{16}$ These values have been included in Tables IX-XII, together with the DFT results reported in the next section. The model used for the different compounds are shown in Fig. 7.

Since the $a b$ initio derivation has produced prejudiceless estimates of the two-body exchange interactions between first and second neighbor centers and of the three four-body 


\begin{tabular}{|c|c|c|c|c|c|}
\hline \multicolumn{6}{|l|}{$-J_{\perp}-J_{\|}$} \\
\hline$\frac{1}{2} J_{\|}-\frac{1}{4}\left(h_{1}+h_{2}-h_{3}\right)$ & $\frac{1}{2} J_{\|}-\frac{1}{4}\left(h_{1}+h_{2}-h_{3}\right)$ & $-J_{\|}-J_{d}$ & & & \\
\hline$\frac{1}{2} J_{\|}-\frac{1}{4}\left(h_{1}+h_{2}-h_{3}\right)$ & $\frac{1}{2} J_{\|}-\frac{1}{4}\left(h_{1}+h_{2}-h_{3}\right)$ & $h_{2}$ & $-J_{\|}-J_{d}$ & & \\
\hline
\end{tabular}

TABLE VIII. The perturbation based effective Hamiltonian on the basis of the model space for $S_{z}=0$ for a rectangular plaquette (for instance, in $\mathrm{SrCu}_{2} \mathrm{O}_{3}$ ).

\begin{tabular}{|c|c|c|c|c|c|}
\hline$\left|a_{\downarrow} b_{\uparrow} c_{\downarrow} d_{\uparrow}\right|$ & $\left|a_{\uparrow} b_{\downarrow} c_{\uparrow} d_{\downarrow}\right|$ & $\left|a_{\downarrow} b_{\downarrow} c_{\uparrow} d_{\uparrow}\right|$ & $\left|a_{\uparrow} b_{\uparrow} c_{\downarrow} d_{\downarrow}\right|$ & $\left|a_{\downarrow} b_{\uparrow} c_{\uparrow} d_{\downarrow}\right|$ & $\left|a_{\uparrow} b_{\downarrow} c_{\downarrow} d_{\uparrow}\right|$ \\
\hline \multicolumn{6}{|l|}{$-J_{\perp}-J_{\|}+\frac{1}{5}\left(h_{1}+h_{2}+h_{3}\right)$} \\
\hline$h_{1}$ & $-J_{\perp}-J_{\|}+\frac{1}{5}\left(h_{1}+h_{2}+h_{3}\right)$ & & & & \\
\hline$\frac{1}{2} J_{\|}-\frac{3}{10}\left(h_{1}+h_{2}\right)+\frac{1}{5} h_{3}$ & $\frac{1}{2} J_{\|}-\frac{3}{10}\left(h_{1}+h_{2}\right)+\frac{1}{5} h_{3}$ & $-J_{\|}-J_{d}+\frac{1}{5}\left(h_{1}+h_{2}+h_{3}\right)$ & & & \\
\hline$\frac{1}{2} J_{\|}-\frac{3}{10}\left(h_{1}+h_{2}\right)+\frac{1}{5} h_{3}$ & $\frac{1}{2} J_{\|}-\frac{3}{10}\left(h_{1}+h_{2}\right)+\frac{1}{5} h_{3}$ & $h_{2}$ & $-J_{\|}-J_{d}+\frac{1}{5}\left(h_{1}+h_{2}+h_{3}\right)$ & & \\
\hline$\frac{1}{2} J_{\perp}-\frac{3}{10}\left(h_{1}+h_{3}\right)+\frac{1}{5} h_{2}$ & $\frac{1}{2} J_{\perp}-\frac{3}{10}\left(h_{1}+h_{3}\right)+\frac{1}{5} h_{2}$ & $\frac{1}{2} J_{d}+\frac{1}{5} h_{1}-\frac{3}{10}\left(h_{2}+h_{3}\right)$ & $\frac{1}{2} J_{d}+\frac{1}{5} h_{1}-\frac{3}{10}\left(h_{2}+h_{3}\right)$ & $-J_{\perp}-J_{d}+\frac{1}{5}\left(h_{1}+h_{2}+h_{3}\right)$ & \\
\hline$\frac{1}{2} J_{\perp}-\frac{3}{10}\left(h_{1}+h_{3}\right)+\frac{1}{5} h_{2}$ & $\frac{1}{2} J_{\perp}-\frac{3}{10}\left(h_{1}+h_{3}\right)+\frac{1}{5} h_{2}$ & $\frac{1}{2} J_{d}+\frac{1}{5} h_{1}-\frac{3}{10}\left(h_{2}+h_{3}\right)$ & $\frac{1}{2} J_{d}+\frac{1}{5} h_{1}-\frac{3}{10}\left(h_{2}+h_{3}\right)$ & $h_{3}$ & $-J_{\perp}-J_{d}+\frac{1}{5}\left(h_{1}+h_{2}+h_{3}\right)$ \\
\hline
\end{tabular}


TABLE IX. Exchange couplings for $\mathrm{La}_{2} \mathrm{CuO}_{4}$ in $\mathrm{meV}\left(h_{2}\right.$ $=h_{3}$ ). per4 and biqua represent, respectively, the extraction using the perturbation-based effective Hamiltonian (Eq. 28) and the biquadratic Heisenberg formulation (Eq. 27).

\begin{tabular}{lccccc}
\hline \hline & & $J$ & $J_{d}$ & $2 h_{1}$ & $2 h_{2}$ \\
\hline CI & per4 & 125 & 7.8 & 14 & 1 \\
\multirow{3}{*}{ B3LYP } & biqua & 124 & 7.0 & 14 & 1 \\
& per4 & 198 & 16 & 64 & \\
$33 \%$ Fock & biqua & 195 & 12.5 & 64 & \\
& per4 & 131 & 6.9 & 20 & \\
& biqua & 130 & 6.0 & 19 & \\
\hline \hline
\end{tabular}

terms, it is possible to check the consistency of the Heisenberg Hamiltonian and the possible deviation from Eqs. (32) and (33).

In all the systems the second-neighbor exchanges $J_{d}$ are antiferromagnetic and much smaller than the first-neighbor interaction, also antiferromagnetic. The $J_{d} / J$ ratio for $\mathrm{La}_{2} \mathrm{CuO}_{4}$ is in good agreement with the square of the ratio of the hopping integrals as determined in a previous work. ${ }^{15}$

The four-body terms $h_{1}$ which imply circulation of the electrons between only nearest-neighbor sites are much larger than the four-body terms $h_{2}$ and $h_{3}$ which arise from the circulation of electrons involving hopping between second neighbors. More quantitatively the ratios $h_{2} / h_{1}$ and $h_{3} / h_{1}$ compare rather well with the ratios $J_{d} / J_{\perp}$ and $J_{d} / J_{\|}$ in agreement with Eqs. (32) and (33) as shown in Table XIII.

\section{B. DFT calculations}

The same systems have been considered to test the validity of the hereafter proposed DFT procedure. In these highly correlated systems one may generate specific self-consistent solutions for each distributions of spin on the four magnetic centers. The number of independent solutions depends on $S_{z}$ being one for $S_{z}=2$, four for $S_{z}=1$, and three for $S_{z}=0$.

For an irregular tetrahedron these eight solutions have different energies and then we have seven energy differences, which is not sufficient to extract the nine amplitudes of the general effective spin operators (four first-neighbor exchanges, two second-neighbor exchanges, and three fourbody terms). When the system presents any symmetry, some energies become identical and also some two-body operators

TABLE X. Exchange couplings for $\mathrm{SrCu}_{2} \mathrm{O}_{3}$ in meV. per4 and biqua represent, respectively, the extraction using the perturbationbased effective Hamiltonian (Eq. 28) and the biquadratic Heisenberg formulation (Eq. 27).

\begin{tabular}{lccccccc}
\hline \hline & & $J_{\|}$ & $J_{\perp}$ & $J_{d}$ & $2 h_{1}$ & $2 h_{2}$ & $2 h_{3}$ \\
\hline CI & per4 & 204.5 & 160 & 16.4 & 34 & 4.1 & 2.7 \\
& biqua & 203 & 157 & 13 & 34 & 4.1 & 2.7 \\
B3LYP & per4 & 247 & 231 & 27 & 120 & & \\
& biqua & 241 & 225 & 21 & 120 & & \\
$33 \%$ Fock & per4 & 166 & 157 & 12 & 39 & & \\
& biqua & 164 & 155 & 10 & 39 & & \\
\hline \hline
\end{tabular}

TABLE XI. Exchange couplings for $\mathrm{CaCu}_{2} \mathrm{O}_{3}$ in meV. per4 and biqua represent, respectively, the extraction using the perturbationbased effective Hamiltonian (Eq. 28) and the biquadratic Heisenberg formulation (Eq. 27).

\begin{tabular}{lccccccc}
\hline \hline & & $J_{\|}$ & $J_{\perp}$ & $J_{d}$ & $2 h_{1}$ & $2 h_{2}$ & $2 h_{3}$ \\
\hline CI & per4 & 148 & 15 & 0.7 & 4 & 1.3 & $\sim 10^{-2}$ \\
& biqua & 147 & 15 & 0.2 & 4 & 1.3 & $\sim 10^{-2}$ \\
B3LYP & per4 & 218 & 20 & 2 & 16 & & \\
& biqua & 217 & 19 & 1 & 16 & & \\
$33 \%$ Fock & per4 & 138 & 13 & 0.85 & 5 & & \\
& biqua & 138 & 13 & 0.6 & 5 & & \\
& & & & & &
\end{tabular}

have equal amplitudes. For an isosceles trapeze, there are two distinct $S_{z}=1$ energies, three $S_{z}=0$ energies, and five energy differences, while four different two-body operators and three four-body terms exist. In this case, an exact extraction of all the parameters is not possible, it is necessary to assume that the four-body operators involving hopping in the diagonal $\left(h_{2}\right.$ and $\left.h_{3}\right)$ are negligible in order to fix the four two-body operators and the dominant four-body term. This is the situation faced in the three-leg ladder $\mathrm{Sr}_{2} \mathrm{Cu}_{3} \mathrm{O}_{5}$ since the bond in the internal leg must be considered as different to the external leg.

For a rectangle, there are just one $S_{z}=1$ energy, three $S_{z}$ $=0$ different solutions and four energy differences. In this case, there are six exchange operators (three two-body operators and three four-body terms). So as in the previous case, it is necessary to neglect the $h_{2}$ and $h_{3}$ terms in order to establish the three two-body amplitudes and the dominant four-body term. These comments are relevant for the two-leg ladders $\left(\mathrm{SrCu}_{2} \mathrm{O}_{3}\right.$ and $\left.\mathrm{CaCu}_{2} \mathrm{O}_{3}\right)$.

For a square plaquette we have one $S_{z}=1$ energy and two $S_{z}=0$ solutions, so three energy differences. There are two two-body amplitudes and three four-body terms. As before, we neglect the $h_{2}$ and $h_{3}$ terms. This is the situation in the $2 \mathrm{D}$ square lattice as $\mathrm{La}_{2} \mathrm{CuO}_{4}$ system.

TABLE XII. Exchange coupling values for $\mathrm{Sr}_{2} \mathrm{Cu}_{3} \mathrm{O}_{5}$ in meV. per4 and biqua represent, respectively, the extraction using the perturbation-based effective Hamiltonian (Eq. 28) and the biquadratic Heisenberg formulation (Eq. 27).

\begin{tabular}{|c|c|c|c|c|c|c|c|}
\hline & & $J_{\|}$ & $J_{\perp}$ & $J_{d}$ & $2 h_{1}$ & $2 h_{2}$ & $2 h_{3}$ \\
\hline \multirow[t]{4}{*}{ CI } & per4 & 197(ext) & 177 & 18 & 39 & 4.1 & 3.3 \\
\hline & & 210 (int) & & & & & \\
\hline & biqua & $195(\mathrm{ext})$ & 177 & 14 & 39 & 4.1 & 3.3 \\
\hline & & 208(int) & & & & & \\
\hline \multirow[t]{4}{*}{ B3LYP } & per4 & $246(\mathrm{ext})$ & 227 & 27 & 110 & & \\
\hline & & 228(int) & & & & & \\
\hline & biqua & $240(\mathrm{ext})$ & 221 & 21 & 120 & & \\
\hline & & 222(int) & & & & & \\
\hline \multirow[t]{4}{*}{$33 \%$ Fock } & per4 & $164($ ext $)$ & 154 & 12 & 37 & & \\
\hline & & 154(int) & & & & & \\
\hline & biqua & $162(\mathrm{ext})$ & 152 & 10 & 40 & & \\
\hline & & 153(int) & & & & & \\
\hline
\end{tabular}



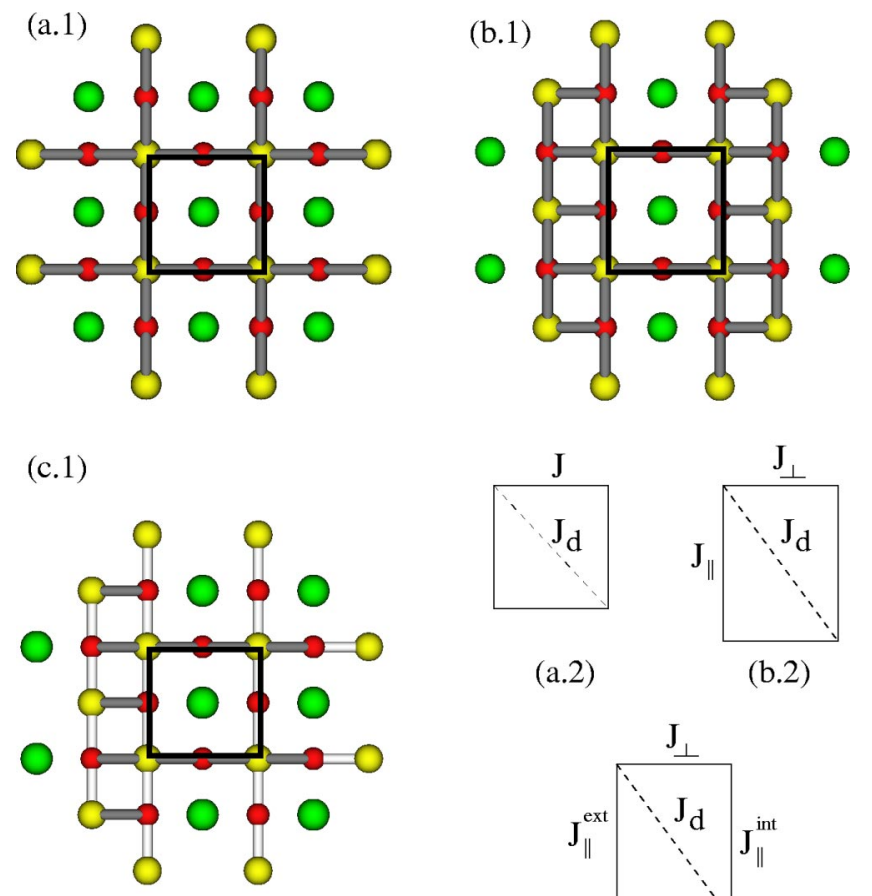

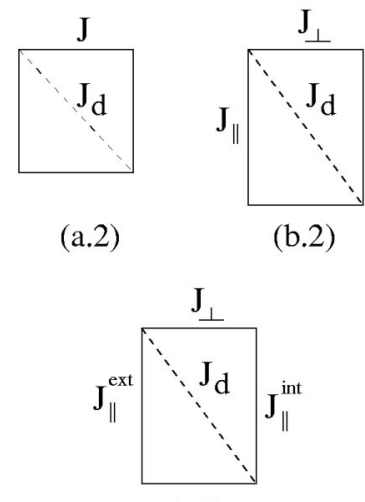

(c.2)

FIG. 7. Models used in ab initio and DFT calculations: $\mathrm{Cu}_{4} \mathrm{O}_{12}$ plaquettes and first-neighbor TIP's environment models for (a1) $\mathrm{La}_{2} \mathrm{CuO}_{4}$, (b1) $\mathrm{SrCu}_{2} \mathrm{O}_{3}$ and $\mathrm{CaCu}_{2} \mathrm{O}_{3}$, and (c1) $\mathrm{Sr}_{2} \mathrm{Cu}_{3} \mathrm{O}_{5}$ compounds. Gray, small black and big dark circles correspond, respectively, to $\mathrm{Cu}, \mathrm{O}$, and counterions atoms $\left(\mathrm{Sr}^{+2}, \mathrm{Ca}^{+2}\right.$, or $\left.\mathrm{La}^{+3}\right)$. Types of exchange interactions in (a2) the $\mathrm{La}_{2} \mathrm{CuO}_{4}$ square plaquette, (b2) the $\mathrm{SrCu}_{2} \mathrm{O}_{3}$ and $\mathrm{CaCu}_{2} \mathrm{O}_{3}$ rectangular plaquette, and (c2) the $\mathrm{Sr}_{2} \mathrm{Cu}_{3} \mathrm{O}_{5}$ irregular rectangular plaquette.

The procedure consists in the identification of the energy of the broken-symmetry solutions with the diagonal elements of the effective Hamiltonian matrix, involving not only the four-body terms as in Tables III and IV and V and VI, but also two-body exchange between first and second neighbors. For instance, in a rectangular plaquette the energy difference between the $S_{z}=2|a(\uparrow) b(\uparrow) c(\uparrow) d(\uparrow)|$ wave function and the $|a(\downarrow) b(\uparrow) c(\uparrow) d(\uparrow)| S_{z}=1$ solution can be identified with

$$
E_{|a(\uparrow) b(\uparrow) c(\uparrow) d(\uparrow)|}-E_{|a(\downarrow) b(\uparrow) c(\uparrow) d(\uparrow)|}=\frac{J_{\perp}+J_{\|}+J_{d}}{2}+\frac{h_{1}}{10}
$$

in the perturbation based effective Hamiltonian and with

$$
E_{|a(\uparrow) b(\uparrow) c(\uparrow) d(\uparrow)|}-E_{|a(\downarrow) b(\uparrow) c(\uparrow) d(\uparrow)|}=\frac{J_{\perp}+J_{\|}+J_{d}}{2}+\frac{h_{1}}{4}
$$

in the Heisenberg extended Hamiltonian. For the three $S_{z}$ $=0$ solutions the energy difference with respect to the quintet state may be identified in the perturbation based effective Hamiltonian as

TABLE XIII. Comparison between the $a b$ initio four-body term ratio and second and first-neighbor exchanges ratio. In all cases the values coming from the biquadratic Heisenberg Hamiltonian have been used.

\begin{tabular}{lcccc}
\hline \hline & $h_{2} / h_{1}$ & $J_{d} / J_{\perp}$ & $h_{3} / h_{1}$ & $J_{d} / J_{\|}$ \\
\hline $\mathrm{SrCu}_{2} \mathrm{O}_{3}$ & 0.12 & 0.08 & 0.08 & 0.07 \\
$\mathrm{Sr}_{2} \mathrm{Cu}_{3} \mathrm{O}_{5}$ & 0.10 & 0.08 & 0.08 & 0.06 \\
$\mathrm{La}_{2} \mathrm{CuO}_{4}$ & 0.07 & 0.05 & 0.07 & 0.05 \\
\hline \hline
\end{tabular}

$$
\begin{aligned}
& E_{|a(\uparrow) b(\uparrow) c(\uparrow) d(\uparrow)|}-E_{|a(\downarrow) b(\uparrow) c(\downarrow) d(\uparrow)|}=J_{\perp}+J_{\|}-\frac{h_{1}}{5}, \\
& E_{|a(\uparrow) b(\uparrow) c(\uparrow) d(\uparrow)|}-E_{|a(\uparrow) b(\downarrow) c(\downarrow) d(\uparrow)|}=J_{\|}+J_{d}-\frac{h_{1}}{5}, \\
& E_{|a(\uparrow) b(\uparrow) c(\uparrow) d(\uparrow)|}-E_{|a(\uparrow) b(\uparrow) c(\downarrow) d(\downarrow)|}=J_{\perp}+J_{d}-\frac{h_{1}}{5} .
\end{aligned}
$$

In the case of the extended Heisenberg Hamiltonian, we have

$$
\begin{aligned}
& E_{|a(\uparrow) b(\uparrow) c(\uparrow) d(\uparrow)|}-E_{|a(\downarrow) b(\uparrow) c(\downarrow) d(\uparrow)|}=J_{\perp}+J_{\|}, \\
& E_{|a(\uparrow) b(\uparrow) c(\uparrow) d(\uparrow)|}-E_{|a(\uparrow) b(\downarrow) c(\downarrow) d(\uparrow)|}=J_{\|}+J_{d}, \\
& E_{|a(\uparrow) b(\uparrow) c(\uparrow) d(\uparrow)|}-E_{|a(\uparrow) b(\uparrow) c(\downarrow) d(\downarrow)|}=J_{\perp}+J_{d} .
\end{aligned}
$$

All the calculations have been performed with hybrid functionals, which mix Fock and Slater exchanges. The original B3LYP mixing ${ }^{26}$ is known to overestimate the delocalization of the magnetic orbitals between the metal and ligands ${ }^{20}$ and to lead to overestimated antiferromagnetic nearest-neighbor exchanges. Better agreement with the experiment is obtained when around a 33\% of Fock exchange is used in the exchange functional. ${ }^{17,19,20}$ It must be noticed that small errors in the $J$ values give strong deviations in the four-body terms, due to the quadratic $J$ dependence of these terms $\left(J_{\text {ring }}=5 J^{2} / U\right)$.

Both kinds of mixing have been used, the results are shown in Tables IX-XII. The models used are the same as in $a b$ initio calculations (Fig. 7). GAUSSIAN $98^{27}$ has been used to perform DFT calculations. ${ }^{28}$

The amplitudes of the parameters are almost identical for the extraction using the perturbation-based effective Hamiltonian and the biquadratic Heisenberg formulation (per4 and biqua, respectively, in Tables IX-XII), the differences being lower than $3 \%$ of error. In all the systems the amplitude of all the exchange operations are overestimated when B3LYP is used. It would be noted that the four spin cyclic term is unlikely large, going up to half of the first neighbor interaction in $\mathrm{SrCu}_{2} \mathrm{O}_{3}$. Increasing the percentage of Fock exchange a better agreement with the CI results is obtained. The fourbody operator also compares quite well with the $a b$ initio estimates. The largest amplitude concerns the $\mathrm{SrCu}_{2} \mathrm{O}_{3}$ system. 


\section{CONCLUSIONS}

For half-filled band, with one electron in one magnetic orbital per site, spin Hamiltonians can be seen as effective Hamiltonians defined in the model space of all neutral valence bond determinants. They may be obtained from the $N$-electron Hamiltonian, for instance, the Hubbard Hamiltonian, through an order-by-order perturbative expansion, in the frame of the quasidegenerate perturbation theory. The two-body exchange operators appear at order one (direct exchange) and two (kinetic exchange). For four-member rings $\overparen{a \rightarrow b \rightarrow c \rightarrow d \rightarrow}$ the fourth-order expansion introduces four-body operators, which commute four spins and corrections to the two-body operators. These corrections result from the cyclic circulation of electrons along the ring. The precise form of these corrections, in terms of $g_{a b c d}=t_{a b} t_{b c} t_{c d} t_{d a} / U^{3}$, have been reported. The so-obtained spin Hamiltonian has been compared with the usual form of the Heisenberg Hamiltonian with biquadratic four-spin operators, and it was shown that this canonical form incorporates four-body cyclic corrections in the two-body exchange. When hopping integrals between second-neighbor atoms are not negligible, two other four-body operators have to be considered.

The amplitudes of the four-body effects have been determined on a series of four-spin lattices, spin ladders with either two or three legs, and the $\mathrm{La}_{2} \mathrm{CuO}_{4}$ square 2D lattice. The knowledge of the eigenenergies and eigenvectors, as obtained from $a b$ initio extended configuration interaction cal- culations, make possible, through the theory of effective Hamiltonians, a prejudiceless evaluation of the two-body exchanges and all four-body effects. The ratios between the amplitudes are consistent with those predicted from the Hubbard Hamiltonian.

This work has also discussed the possibility to evaluate the amplitude of four-body cyclic effects from DFT symmetry-broken energies. The information is not sufficient for an evaluation of all the amplitudes, and one has to suppose some of the terms to be negligible. The reliability of the results dramatically depends on the exchange potential employed. The popular B3LYP which is known to overestimate the first-neighbor magnetic coupling by a factor between 1.5 and 2, leads to unreasonably large values of the dominant four-body effect. Increasing to $33 \%$ the Fock component of the hybrid exchange not only provides satisfactory values of the nearest-neighbor coupling constant but also produces a reliable estimate of the leading four-body term.

\section{ACKNOWLEDGMENTS}

The authors acknowledge the support of a French-Catalan international cooperation project (Grant No. PICS 1458) and of a special funding by the CNRS to support European program COST No. 026/0008/02. The laboratoire de Physique Quantique is Unite Mixte de Recherche (UMR 5626) of the CNRS.
*Email address: calzado@us.es

†Email address: malrieu@irsamc.ups-tlse.fr

${ }^{1}$ W. Heisenberg, Z. Phys. 49, 619 (1928).

${ }^{2}$ P.A.M. Dirac, Proc. R. Soc. London, Ser. A 123, 714 (1929); The Principles of Quantum Mechanics (Clarendon, Oxford, 1947).

${ }^{3}$ J.H. Van Vleck, The Theory of Electric and Magnetic Susceptibilities (Oxford University Press, Oxford, 1932).

${ }^{4}$ J.-P. Malrieu and D. Maynau, J. Am. Chem. Soc. 104, 3021 (1982).

${ }^{5}$ A.H. MacDonald, S.M. Girvin, and D. Yoshioka, Phys. Rev. B 37, 9753 (1988).

${ }^{6}$ M. Takahashi, J. Phys. C 10, 1289 (1977).

${ }^{7}$ M. Roger and J.M. Delrieu, Phys. Rev. B 39, 2299 (1989).

${ }^{8}$ H. Schmidt and Y. Kuramoto, Physica C 167, 263 (1990).

${ }^{9}$ Y. Honda, Y. Kuramoto, and T. Watanabe, Phys. Rev. B 47, 11329 (1993).

${ }^{10}$ J. Lorenzana, J. Eroles, and S. Sorella, Phys. Rev. Lett. 83, 5122 (1999).

${ }^{11}$ J. Eroles, C.D. Batista, S.B. Bacci, and E.R. Gagliano, Phys. Rev. B 59, 1468 (1999)

${ }^{12}$ S. Brehmer, H.J. Mikeska, and M. Müller, Phys. Rev. B 60, 329 (1999).

${ }^{13}$ M. Matsuda, K. Katsumata, R.S. Eccleston, S. Brehmer, and H.-J. Mikeska, Phys. Rev. B 62, 8903 (2000).

${ }^{14}$ C.J. Calzado and J.-P. Malrieu, Eur. Phys. J. B 21, 375 (2001).

${ }^{15}$ C.J. Calzado and J.-P. Malrieu, Phys. Rev. B 63, 214520 (2001).

${ }^{16}$ C.J. Calzado, C. de Graaf, E. Bordas, R. Caballol, and J.-P. Malrieu, Phys. Rev. B 67, 132409 (2003).

${ }^{17}$ R.L. Martin and F. Illas, Phys. Rev. Lett. 79, 1539 (1997); F. Illas and R.L. Martin, J. Chem. Phys. 108, 2519 (1998).

${ }^{18}$ C. Adamo, V. Barone, A. Bencini, F. Totti, and I. Ciofini, Inorg. Chem. 38, 1996 (1999).

${ }^{19}$ C.J. Calzado, J. Cabrero, J.P. Malrieu, and R. Caballol, J. Chem. Phys. 116, 3985 (2002).

${ }^{20}$ J. Cabrero, C.J. Calzado, D. Maynau, R. Caballol, and J.P. Malrieu, J. Phys. Chem. A 106, 8146 (2002).

${ }^{21}$ E. Ruiz, J. Cano, S. Alvarez, and P. Alemany, J. Comput. Chem. 20, 1391 (1999).

${ }^{22}$ C. Bloch, Nucl. Phys. 6, 329 (1958).

${ }^{23}$ J. des Cloizeaux, Nucl. Phys. 20, 321 (1960).

${ }^{24}$ For sake of simplicity and generality, we call tetrahedron the graph of four summits and six bonds joining the summits two by two.

${ }^{25}$ L. Noodleman and J.G. Norman, Jr., J. Chem. Phys. 70, 4903 (1979); L. Noodleman, ibid. 74, 5737 (1981); L. Noodleman and E.R. Davidson, Chem. Phys. 109, 131 (1986); L. Noodleman, C.Y. Peng, D.A. Case, and J.M. Mouesca, Coord. Chem. Rev. 144, 199 (1995).

${ }^{26} \mathrm{~B} 3 \mathrm{LYP}$ is the exchange-correlation functional proposed by Becke [J. Chem. Phys. 98, 5648 (1993)]. This functional results from a mixing of the Fock $(20 \%)$ and local Slater $(80 \%)$ exchange, and the nonlocal Becke exchange correction. In the correlation part, the local Vosko, Wilk, and Nusair functional and the nonlocal Lee, Yang, and Parr correlation correction have been used.

${ }^{27}$ M.J. Frisch, G.W. Trucks, H.B. Schlegel, G.E. Scuseria, M.A. Robb, J.R. Cheeseman, V.G. Zakrzewski, J.A. Montgomery, Jr., R.E. Stratmann, J.C. Burant, S. Dapprich, J.M. Millam, A.D. Daniels, K.N. Kudin, M.C. Strain, O. Farkas, J. Tomasi, V. Bar- 
one, M. Cossi, R. Cammi, B. Mennucci, C. Pomelli, C. Adamo, S. Clifford, J. Ochterski, G.A. Petersson, P.Y. Ayala, Q. Cui, K. Morokuma, N. Rega, P. Salvador, J.J. Dannenberg, D.K. Malick, A.D. Rabuck, K. Raghavachari, J.B. Foresman, J. Cioslowski, J.V. Ortiz, A.G. Baboul, B.B. Stefanov, G. Liu, A. Liashenko, P. Piskorz, I. Komaromi, R. Gomperts, R.L. Martin, D.J. Fox, T. Keith, M.A. Al-Laham, C.Y. Peng, A. Nanayakkara, M. Challacombe, P.M.W. Gill, B. Johnson, W. Chen, M.W. Wong, J.L. Andres, C. Gonzalez, M. Head-Gordon, E.S. Replogle, and J.A. Pople, Gaussian98, Revision A.11.3, Gaussian, Inc., Pittsburgh, PA, 2002.

${ }^{28}$ In all the calculations the basis set and pseudopotential of Hay and Wadt for $\mathrm{Cu}$ atoms have been used [P.J. Hay and W.R. Wadt, J. Chem. Phys. 82, 284 (1985)]. For oxygen atoms an all electron basis set $(10 s 5 p)$ contracted to $[3 s 2 p]$ is employed. In the case of the bridging oxygen atoms a polarization $d$ function has also been included [T.H. Dunning, Jr., ibid. 53, 2823 (1970); T.H. Dunning, Jr. and P.J. Hay, in Methods of Electronic Structure Theory, edited by H.F. Schaefer III (Plenum Press, New York, 1977), Vol. 2]. In order to model the infinite lattice, the first-shell of neighbors has been replaced by pseudopotential, which incorporate both electrostatic and exclusion effects of these ions. The Madelung field of the remote atoms has been considered by means of point charges. 